\title{
Effects of angiotensin II on transient receptor potential melastatin 7 channel function in cardiac fibroblasts
}

\author{
YANLI ZHOU $^{1 *}, \mathrm{XIN} \mathrm{YI}^{1 *}, \mathrm{TENG} \mathrm{WANG}^{2}$ and MINGJIANG LI ${ }^{1}$ \\ ${ }^{1}$ Department of Cardiology; ${ }^{2}$ Gladstone Institute of Cardiovascular Disease, \\ Renmin Hospital of Wuhan University, Wuhan, Hubei 430060, P.R. China
}

Received June 2, 2014; Accepted February 19, 2015

DOI: 10.3892/etm.2015.2362

\begin{abstract}
The aim of the present study was to investigate the electrophysiological properties of transient receptor potential melastatin 7 (TRPM7) channels in the cell membranes of cardiac fibroblasts (CFs). CFs obtained from Sprague-Dawley rats were treated with six different concentrations of angiotensin (Ang) II for $12 \mathrm{~h}$. The optimum concentration of Ang II for inducing fibrosis was selected by measuring collagen protein expression levels at each concentration. The optimum concentration of Ang II was then used to treat the CFs at five different time-points, and the TRPM7 current across the CF membranes was recorded along with the collagen protein levels in each group. The optimum Ang II concentration for inducing fibrosis was found to be $1 \mathrm{nmol} / \mathrm{l}$; when it was administered to CFs at $0,6,12,24$ and $48 \mathrm{~h}$, the protein expression levels of TRPM7 and collagen III initially increased, reaching maximum levels at $12 \mathrm{~h}$, and then decreased. This result positively correlated with the TRPM7 current levels on either side of the CF membranes: The TRPM7 current under the effect of the optimal Ang II concentration for inducing fibrosis was initially increased and then later decreased. This is one of the key mechanisms by which Ang II mediates the proliferation and apoptosis of CFs. This knowledge may be used to identify treatments for the prevention of cardiac fibrosis.
\end{abstract}

\section{Introduction}

Cardiovascular diseases have become the leading cause of disability and mortality worldwide (1). Cardiac fibrosis is an important pathological and physiological response to various diseases (at the middle and end stages), including heart failure,

Correspondence to: Dr Mingjiang Li, Department of Cardiology, Renmin Hospital of Wuhan University, 238 Jiefang Road, Wuhan, Hubei 430060, P.R. China

E-mail: 754851539@qq.com

*Contributed equally

Key words: transient receptor potential melastatin 7, angiotensin II, cardiac fibrosis hypertrophic cardiomyopathy, myocardial infarction and malignant arrhythmias, and it is a challenge in current clinical therapy (1). A growing body of evidence indicates that cardiac fibroblasts (CFs) are the main cells responsible for the cardiac fibrosis process. Under the impact of a variety of cell stimulating factors, CFs promote an increasing influx of external ions. This leads to pathologic hyperplasia and pathological differentiation, the formation of cellular matrix-like collagen and the initiation of the tissue fibrosis process. Therefore, the identification of methods to enable early intervention in the function of CFs, in order to block the onset and delay the development of cardiac fibrosis, has become a key issue for study in recent decades $(2,3)$.

A type of $\mathrm{Ca}^{2+}$-permeable channel with a high expression level of transient receptor potential melastatin 7 (TRPM7) has been found in the cell membranes of Sprague-Dawley (SD) rats $(4,5)$. Previous studies have shown that TRPM7 is the only calcium channel expressed on the cell membrane of CFs (6) and that $\mathrm{Ca}^{2+}$ signaling is closely associated with the initiation of fibrosis (7-9). These findings strongly suggest that TRPM7 may be the one of the most potent fibrosis factors, playing an important role in the molecular mechanism and pathological and physiological processes by which angiotensin II (Ang II) induces cardiac fibrosis. Therefore, it is hypothesized that TRPM7 is expressed at high levels when stimulated by Ang II. $\mathrm{Ca}^{2+}$ signaling on the cell membrane of CFs may be mediated by the stimulatory effect of Ang II on TRPM7, causing collagen synthesis in the CFs to increase and leading to the fibrosis of cardiac interstitial tissue.

\section{Materials and methods}

Subjects. SD rats ( $<3$ days old) were purchased from Wuhan University Center for Animal Experiment/Animal Biological Safety Level-3 Laboratory (Wuhan, China). The study was approved by the Medical Ethics Committee of Renmin Hospital of Wuhan University.

Cell culture. The tissue method of Runnels et al (10) was adopted to separate and collect the rat CFs. Following resuspension in the culture solution [Dulbecco's modified Eagle's medium (DMEM)-F12 (Invitrogen Life Technologies, Carlsbad, CA, USA) supplemented with $10 \%$ fetal bovine serum (Santa Cruz Biotechnology, Inc., Dallas, TX, USA)], CFs were collected using the method of differential sedimentation and inoculated in 
a culture dish. The cells were then synchronized by the starvation method in fetal bovine serum-free medium and placed in an incubator for subculture at $37^{\circ} \mathrm{C}$ and $5 \% \mathrm{CO}_{2}$. The culture solution (containing mycillin and DMEM-F-12] was replaced once every $36 \mathrm{~h}$. When the cell density was $\geq 90 \%$, subculture was conducted by digesting cells with $0.25 \%$ trypsin and $0.5 \mathrm{mM}$ EDTA. One agar plate was retained for the Ang II treatment group and the establishment of the normal control group.

\section{Cell interventions}

Determination of the concentration of Ang II having the optimal fibrosis-inducing effect. i) Treatment. The cultured CFs were separated into six groups, and DMEM culture solution (containing 10\% fetal calf serum) was added along with $0,1,5$, 10,100 or $1,000 \mathrm{nmol} / 1$ Ang II. After $12 \mathrm{~h}\left(37^{\circ} \mathrm{C}\right)$, the expression levels of collagen I and collagen III proteins in each group were measured by western blotting and the group in which the concentration of Ang II had the greatest inducement effect for fibrosis was selected.

ii) Western blotting. Western blotting technology was adopted to evaluate the expression levels of collagen I and collagen III proteins in each group. The total cellular protein was extracted using a protein extraction kit in accordance with the manufacturer's instructions (Beyotime Institute of Biotechnology, Wuhan, China). The Bradford method was used to determine the protein concentration and equalize the protein concentrations in the samples of the different groups through adjustment. Electrophoresis by the SDS-PAGE technique was conducted based on $50 \mu \mathrm{g}$ total protein/lane loading using electrophoresis equipment from Beijing Liuyi Instrument Factory (Beijing, China) and an SDS-PAGE gel preparation regulator from Wuhan Huge Biotechnology Co., Ltd. (Wuhan, China). Following the electrophoresis, the protein on the gel was transferred onto a polyvinylidene fluoride membrane. The gel was sealed with $5 \%$ skimmed milk powder [prepared with $0.5 \%$ Tris-buffered saline-Tween ${ }^{\circledR}$ (TBST)] at room temperature for $1 \mathrm{~h}$. Rabbit polyclonal antibodies (1:500; Abcam, Cambridge, UK) against collagen I (cat. no. ab34710) and collagen III (cat. no. ab7778) were added following membrane rinsing and the membrane was incubated at $4{ }^{\circ} \mathrm{C}$ overnight. The membrane was then incubated at room temperature for $30 \mathrm{~min}$ with goat anti-rabbit secondary antibody (1:3,000; cat. no. 074-1506; KPL, Inc., Gaithersburg, MD, USA) and washed three times (5 min each time) in a gyratory shaker at room temperature with TBST. Finally, fixation and color development with 3,3'-diaminobenzidine was conducted in a dark chamber. The conditions of exposure were adjusted based on the luminous intensity; the photographic film was scanned and filed; and AlphaEaseFC software (Alpha Innotech, San Leandro, CA, USA) was used to analyze the optical density of the blots.

Recording the CF TRPM7 current. i) Treatment. The cultured CFs were separated into five groups and treated for $0,6,12,24$ and $48 \mathrm{~h}$ with the optimal concentration of Ang II for inducing fibrosis. The TRPM7 current was determined for the five time-points as described below. In addition, the collagen and TRPM7 protein levels of the cells were determined using the previously described western blotting technique (anti-TRPM7; cat. no. ab109438; Abcam).

ii) Electrophysiological recording. The Axopatch 200B amplifier (Molecular Devices, LLC, Sunnyvale, CA, USA) was used to record the whole-cell currents of the CFs. The ramp stimulus procedure was adopted with a stimulating voltage of $120 \pm 100 \mathrm{mV}$. The internal fluid (11) in the electrode included: $145 \mathrm{mmol} / 1$ cesium mesilate; $8 \mathrm{mmol} / \mathrm{l}$ sodium chloride; $10 \mathrm{mmol} / \mathrm{l}$ ethylene glycol tetra-acetic acid and $10 \mathrm{mmol} / \mathrm{l}$ hydroxyethylpiperazine ethane sulfonic acid (Sinopharm, Shanghai, China). The $\mathrm{pH}$ value was adjusted to 7.2 with sodium hydroxide solution. In certain experiments, $3 \mathrm{mmol} / \mathrm{M} \mathrm{Mg}^{2+}$ was added to the internal fluid (calculated by MaxChelator software; http://maxchelator.stanford.edu). The standard extracellular Tyrode solution was composed of $140 \mathrm{mmol} / 1$ sodium chloride, $5 \mathrm{mmol} / 1$ potassium chloride, $2 \mathrm{mmol} / 1$ calcium chloride, $20 \mathrm{mmol} / 1$ hydroxyethylpiperazine ethane sulfonic acid and $10 \mathrm{mmol} / \mathrm{l}$ glucose (Sinopharm). The $\mathrm{pH}$ value was adjusted to 7.4 with sodium hydroxide solution.

Statistical analysis. Data are presented as the mean \pm standard deviation. Comparisons among groups were conducted with a Student's t-test on two independent samples. Statistical analysis was conducted using SPSS software (version 16.0; SPSS Inc., Chicago, IL, USA). P<0.05 was considered to indicate a statistically significant difference.

\section{Results}

Microscopic examination of the cultured CFs. During microscopic examination, the CFs were observed to be elongated and spindle-like. They grew with a homogeneous distribution and arranged themselves into garland-like or roller forms. No cardiac impulse was observed (Fig. 1).

Protein expression levels in CFs following treatment for $12 \mathrm{~h}$ with Ang II at various concentrations. The expression levels of collagen I and collagen III in the CFs treated for $12 \mathrm{~h}$ with Ang II at different concentrations were determined by western blotting. Analysis of the results indicated that the expression levels of the two types of collagen induced by Ang II (1 nmol/l) were increased significantly compared with those in the other groups $(\mathrm{P}<0.05)$, which indicated that the concentration of Ang II having the best inducement effect for fibrosis was $1 \mathrm{nmol} / \mathrm{l}$ (Fig. 2).

Protein expression levels of collagen I, collagen III and TRPM7 in CFs following treatment with Ang II for different time periods. The protein expression levels of collagen III and TRPM7 gradually increased over time and reached a maximum when the duration of treatment was $12 \mathrm{~h}$, gradually decreasing subsequently (Fig. 3). The TRPM7 current underwent corresponding changes (gradually increasing and then decreasing with time) and reached a maximum (8-fold higher than that of the $0 \mathrm{~h}$ group) when the duration of treatment was $12 \mathrm{~h}$ (as shown in Fig. 4). The protein expression level of collagen III was lower than that of the control group when the duration of intervention was $48 \mathrm{~h}(\mathrm{P}<0.05)$. The protein expression level of TRPM7 remained higher than that of the control group $(\mathrm{P}<0.05)$. The protein expression level of collagen I reached a maximum when the duration of intervention was $24 \mathrm{~h}$, and was significantly different from that in the other groups $(\mathrm{P}<0.05)$. 


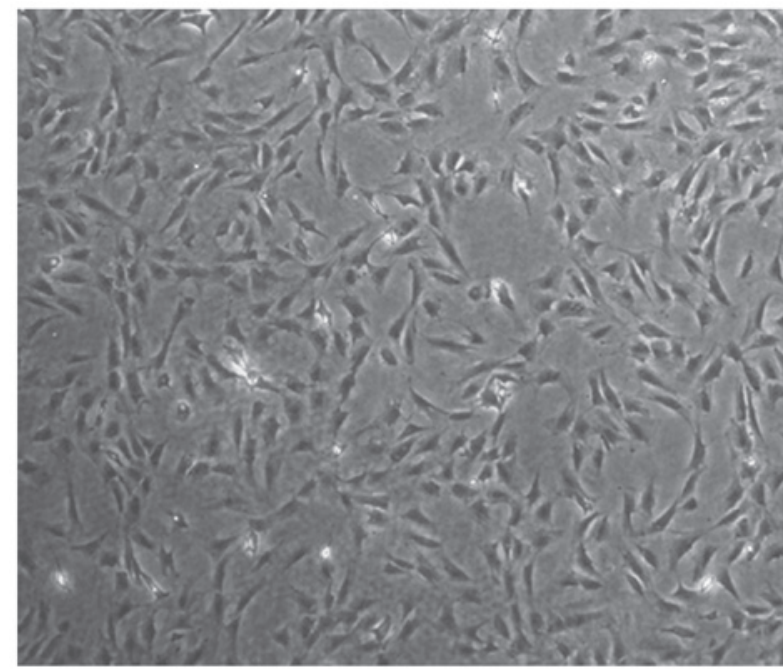

Figure 1. Cultured cardiac fibroblasts (magnification, $\mathrm{x} 40$ ).

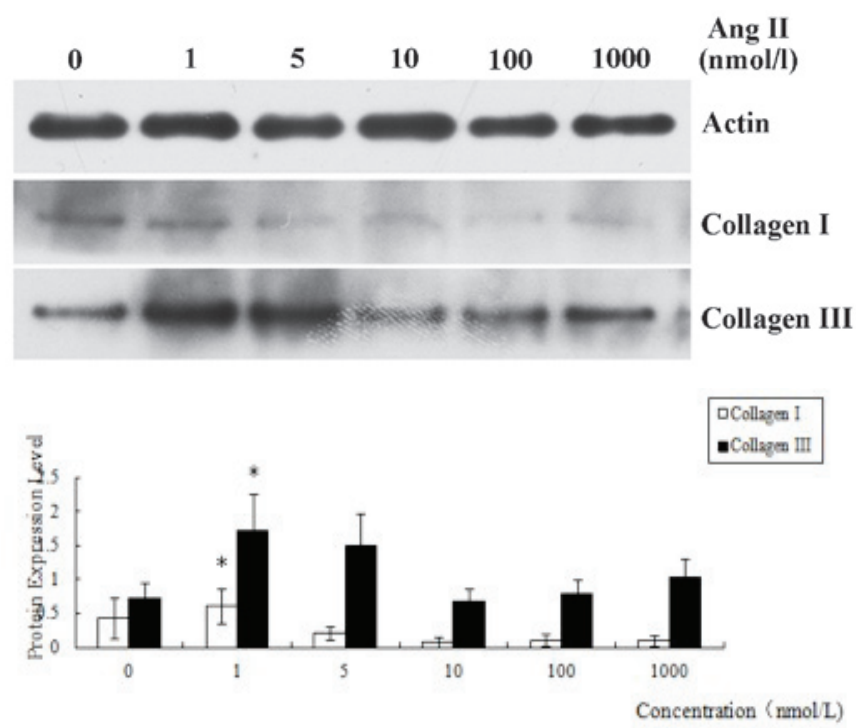

Figure 2. Protein expression levels of collagen I and collagen III obtained by treating cardiac fibroblasts for $12 \mathrm{~h}$ with angiotensin (Ang) II at various concentrations. $\beta$-actin was used as a control. ${ }^{*} \mathrm{P}<0.05$ vs. $0,10,100$ and $1000 \mathrm{nmol} / \mathrm{l}$ groups.

\section{Discussion}

TRPM7, belonging to the TRP superfamily, is an ion channel with dual channel and kinase activity (10). Its expression enables inward rectifier currents of $\mathrm{Ca}^{2+}$ to permeate cell membranes. The current amplitude rises with the consumption of $\mathrm{Mg}^{2+}$ inside the cell and is inhibited by other bivalent ions. TRPM7 is activated by protein kinase A and deactivated by phosphatidylinositol 4,5-bisphosphate (10). Previous studies have shown that TRPM7 plays a significant role in the growth and apoptosis of histiocytes, is activated by mechanical stress and is associated with vasodilation (12-14). It has been reported that the expression of TRPM7 by vascular smooth muscle cells is significantly upregulated when activated by transforming growth factor (TGF)- $\beta 1$ and fluid flow (15). The expression of TRPM7 in CFs has previously been verified by immune cytochemistry (16). Two

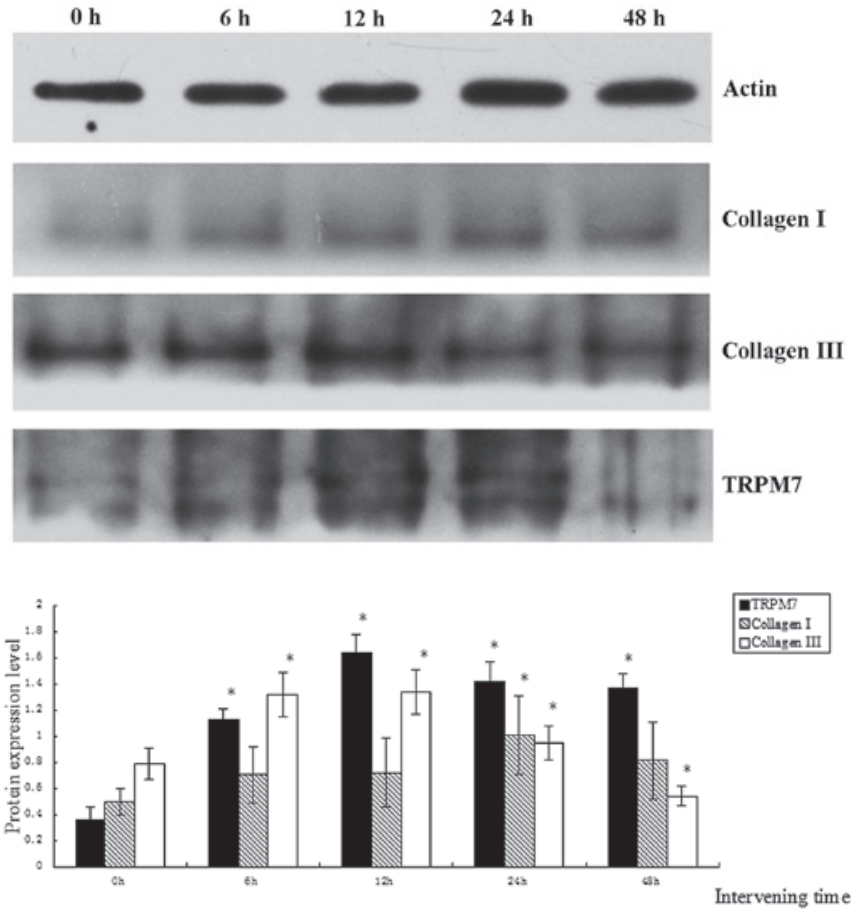

Figure 3. Comparison of protein expression levels of collagen I, collagen III and TRPM7 obtained by treating cardiac fibroblasts for $0,6,12,24$ and $48 \mathrm{~h}$ with angiotensin II $(1 \mathrm{nmol} / \mathrm{l})$. $\mathrm{P}<0.05$. TRPM, transient receptor potential melastatin.

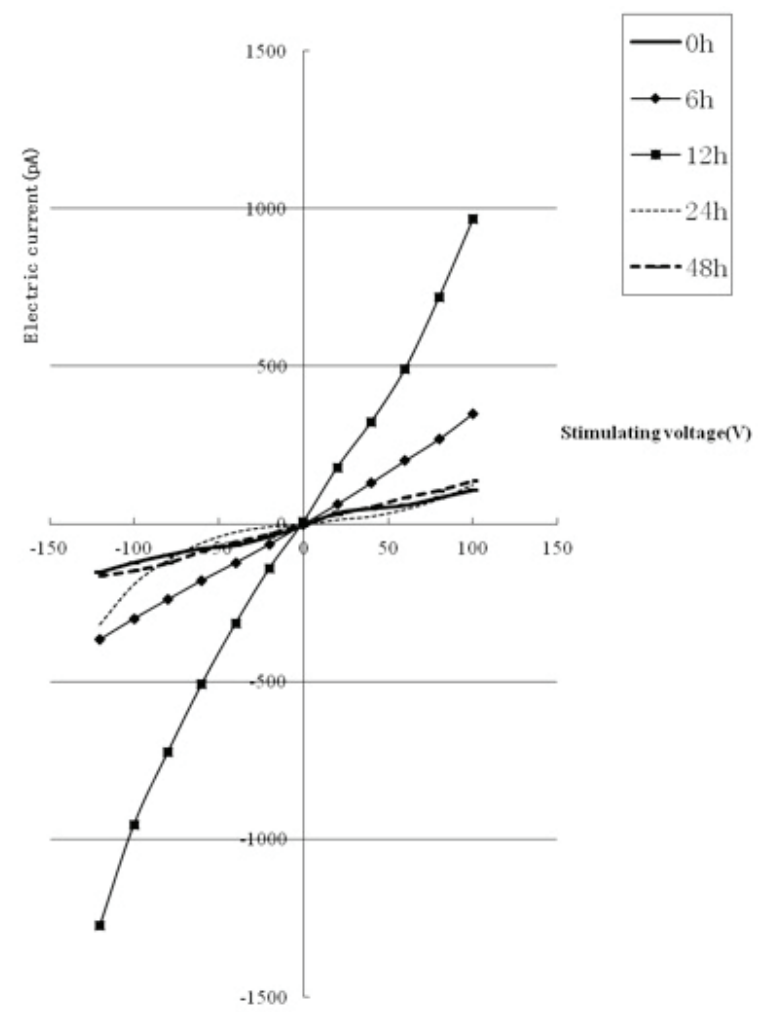

Figure 4. TRPM7 current level obtained by treating cardiac fibroblasts for 0 , $6,12,24$ and $48 \mathrm{~h}$ with angiotensin II ( $1 \mathrm{nmol} / \mathrm{l})$. TRPM, transient receptor potential melastatin.

characteristics of TRPM7 channels, specifically, that they are non-voltage-gated and have $\mathrm{Ca}^{2+}$ permeability, suggest 
that they may have significant pathological and physiological functions in various cells, particularly in non-excitable cells such as CFs.

Preliminary research suggested that the TRPM7 ion channel is the molecular basis of the TRPM currents observed in our previous studies $(4,5)$. It also suggested that the resting membrane potential level of CFs is $-25 \mathrm{mV}$, under which all voltage-gated channels are in the deactivation state. This further supports TRPM7 being the only channel for the inward flow of $\mathrm{Ca}^{2+}$ in CFs. Under the impact of endogenous and exogenous stimulating factors, TRPM7 channels would open, leading to a considerable flow of extracellular $\mathrm{Ca}^{2+}$ into the cell. This flow is likely to cause collagen synthesis to increase, leading to fibrosis. Furthermore, the considerable inward flow of $\mathrm{Ca}^{2+}$ would activate signaling pathways such as the mitogen-activated protein kinase (MAPK) and TGF- $\beta /$ Smad pathways, causing inflammation, apoptosis and cellular differentiation leading to an acceleration of fibrosis.

During one preliminary clinical study, it was found that the myocardial tissues of patients suffering from atrial fibrillation had a certain degree of fibrosis (17); another preliminary clinical study showed that in the myocardial tissues of patients suffering from atrial fibrillation due to rheumatic heart disease, the mRNA and protein expression levels of TRPM7 rose significantly compared with those of individuals with sinus rhythm (18). These studies suggest that under the impact of abnormal stimulation, such as an increased volume and pressure load, TRPM7, the only $\mathrm{Ca}^{2+}$ channel protein on CFs would be activated and its expression increased. This would increase the internal flow of extracellular $\mathrm{Ca}^{2+}$ to the cell to activate and launch biological signaling cascades leading to the pathological transfer, differentiation and secretion of a large quantity of extracellular matrix, finally giving rise to cardiac interstitial fibrosis.

The primary causes of fibrosis include the disequilibrium between pro-fibrogenic cell growth factors and anti-fibrogenic cell growth factors. Studies on myocardial fibrosis have gradually focused on cell growth factors, which include TGF, connective tissue growth factor, basic fibroblast growth factor and hepatocyte growth factor $(8,19,20)$. Ang II, frequently researched in recent years, is the pro-fibrogenic cell growth factor (8). Ang II plays a significant role in the occurrence and development of organ fibrosis. Through protein quantification, the present study verified that the concentration of Ang II having the greatest inducement effect for fibrosis was $1 \mathrm{nmol} / \mathrm{l}$. The results also showed that Ang II at various concentrations had different effects on the growth of CFs: the effect of Ang II on CFs was concentration-dependent. The effect on the growth of CFs decreased as the concentration continued to rise; this may be associated with an increase in the induction of apoptosis and necrosis of the cells. The protein expression levels of TRPM7 and collagen III obtained by treating CFs for 0, 6, 12, 24 and $48 \mathrm{~h}$ with the optimum concentration of Ang II increased at first and reached a maximum when the duration of intervention was $12 \mathrm{~h}(\mathrm{P}<0.05)$ and then decreased gradually. The fact that the inward and outward currents of TRPM7 recorded on the CF cell membranes also took on corresponding trends in changes (first increasing and then decreasing) showed that the influence of Ang II on the cell is time-dependent to some extent. In the present study, the protein expression of collagen I also took on the same change trend (first increasing and then decreasing), but it reached a maximum when the duration of intervention was $24 \mathrm{~h}$, and then the expression decreased and took on the same change trend as was observed for the other two proteins.

In conclusion, the present study demonstrated that under the effect of Ang II at the concentration that had the maximum inducement effect on fibrosis, the protein expression level of TRPM7 by the CFs increased, the internal flow of $\mathrm{Ca}^{2+}$ increased and collagens I and III were expressed, promoting fibrosis. When the exposure to Ang II was prolonged, the induction of CFs to undergo apoptosis and necrosis increased, which may have given rise to various inflammatory reactions and further promoted fibrosis. The limitation of this study lies in that the experiment was conducted in vitro; therefore, it is necessary to conduct in vivo experiments with CFs to further verify the hypothesis that Ang II leads to cardiac fibrosis by mediating TRPM7 on the CF cell membrane.

\section{Acknowledgements}

This study was financially supported by the National Natural Science Grant of China (No. 81170085). The authors are grateful to the staff members at their institutions for their valuable comments.

\section{References}

1. Brown RD, Ambler SK, Mitchell MD and Long CS: The cardiac fibroblast: therapeutic target in myocardial remodeling and failure. Annu Rev Pharmacol Toxicol 45: 657-687, 2005.

2. Camelliti P, Borg TK and Kohl P: Structural and functional characterisation of cardiac fibroblasts. Cardiovasc Res 65: 40-51, 2005.

3. Pawlinski R, Fernandes A, Kehrle B, et al: Tissue factor deficiency causes cardiac fibrosis and left ventricular dysfunction. Proc Natl Acad Sci USA 99: 15333-15338, 2002.

4. Jiang J, Li M and Yue L: Potentiation of TRPM7 inward currents by protons. J Gen Physiol 126: 137-150, 2005.

5. Li M, Jiang J and Yue L: Functional characterization of homoand heteromeric channel kinases TRPM6 and TRPM7. J Gen Physiol 127: 525-537, 2006.

6. Zhang YH, Sun HY, Chen KH, Du XL, Liu B, Cheng LC, Li X, Jin MW and Li GR: Evidence for functional expression of TRPM7 channels in human atrial myocytes. Basic Res Cardiol 107: 282, 2012.

7. González A, López B and Díez J: Fibrosis in hypertensive heart disease: role of the renin-angiotensin-aldosterone system. Med Clin North Am 88: 83-97, 2004.

8. Olson ER, Shamhart PE, Naugle JE and Meszaros JG: Angiotensin II-induced extracellular signal-regulated kinase $1 / 2$ activation is mediated by protein kinase Cdelta and intracellular calcium in adult rat cardiac fibroblasts. Hypertension 51: 704-711, 2008.

9. Manabe I, Shindo T and Nagai R: Gene expression in fibroblasts and fibrosis: Involvement in cardiac hypertrophy. Circ Res 91: 1103-1113, 2002.

10. Runnels LW, Yue L and Clapham DE: The TRPM7 channel is inactivated by PIP (2) hydrolysis. Nat Cell Biol 4: 329-336, 2002.

11. Jiang J, Li M and Yue L: Potentiation of TRPM7 inward currents by protons. J Gen Physiol 126: 137-150, 2005.

12. Baldoli E, Castiglioni S and Maier JA: Regulation and function of TRPM7 in human endothelial cells: TRPM7 as a potential novel regulator of endothelial function. PLoS One 8: e59891, 2013.

13. Chen YF, Chen YT, Chiu WT and Shen MR: Remodeling of calcium signaling in tumor progression. J Biomed Sci 20: 23, 2013.

14. Simon F, Varela D and Cabello-Verrugio C: Oxidative stress-modulated TRPM ion channels in cell dysfunction and pathological conditions in humans. Cell Signal 25: 1614-1624, 2013. 
15. Park HS, Hong C, Kim BJ and So I: The pathophysiologic roles of TRPM7 channel. Korean J Physiol Pharmacol 18: 15-23, 2014.

16. Yue Z, Zhang Y, Xie J, Jiang J and Yue L: Transient receptor potential (TRP) channels and cardiac fibrosis. Curr Top Med Chem 13: 270-282, 2013

17. Yi X, Li M, Ma L, et al: Relationship of CTGF, HGF and atrial fibrosis in rheumatic heart disease patients with atrial fibrillation. Wuhan Da Xue Xue Bao. Yi Xue Ban 33: 824-828, 2012 (In Chinese).

18. Li M, Yi X, Ma L, et al: Relationship of TRPM7 and atrial fibrosis in rheumatic heart disease patients with atrial fibrillation. Wuhan Da Xue Xue Bao. Yi Xue Ban 33: 875-878, 2012 (In Chinese).
19. Yi X, Li X, Zhou Y, et al: Hepatocyte growth factor regulates the TGF- $\beta 1$-induced proliferation, differentiation and secretory function of cardiac fibroblasts. Int J Mol Med 34: 381-390, 2014

20. Kinoshita T, Ishikawa Y, Arita M, et al: Antifibrotic response of cardiac fibroblasts in hypertensive hearts through enhanced TIMP-1 expression by basic fibroblast grow th factor. Cardiovasc Pathol 23: 92-100, 2014. 